\title{
Multimodulus Blind Equalization Algorithm Using Oblong QAM Constellations for Fast Carrier Phase Recovery
}

\author{
Jenq-Tay Yuan ${ }^{1}$ and Tzu-Chao Lin ${ }^{2}$ \\ Graduate Institute of Applied Science and Engineering, Fu Jen Catholic University \\ Taipei 24205, Taiwan, R.O.C.
}

\section{Introduction}

Adaptive channel equalization without a training sequence is known as blind equalization [1]-[11]. Consider a complex baseband model with a channel impulse response of $c_{n}$. The channel input, additive white Gaussian noise, and equalizer input are denoted by $s_{n}, w_{n}$ and $u_{n}$, respectively, as shown in Fig. 1. The transmitted data symbols, $s_{n}$, are assumed to consist of stationary independently and identically distributed (i.i.d.) complex non-Gaussian random variables. The channel is possibly a non-minimum phase linear time-invariant filter. The equalizer input, $u_{n}=s_{n} * c_{n}+w_{n}$ is then sent to a tap-delay-line blind equalizer, $f_{n}$, intended to equalize the distortion caused by inter-symbol interference (ISI) without a training signal, where $*$ denotes the convolution operation. The output of the blind equalizer, $y_{n}=f_{n}^{\star} * u_{n}=s_{n} * h_{n}+f_{n}^{\star} * w_{n}$, can be used to recover the transmitted data symbols, $s_{n}$, where $\star$ denotes complex conjugation and $h_{n}=f_{n}^{\star} * c_{n}$ denotes the impulse response of the combined channel-equalizer system whose parameter vector can be written as the time-varying vector $\mathbf{h}_{n}=\left[h_{n}(1), h_{n}(2), \ldots\right]^{T}$ with $M$ arbitrarily located non-zero components at a particular instant, $n$, during the blind equalization process, where $M=1,2,3, \ldots$. For example, if $M=3$ and $I_{M}=\{1,2,5\}$ is any $M$-element subset of the integers, then $\mathbf{h}_{n}=\left[h_{n}(1), h_{n}(2), 0,0, h_{n}(5), 0 \ldots 0\right]^{T}$ is a representative value of $\mathbf{h}_{n}$.

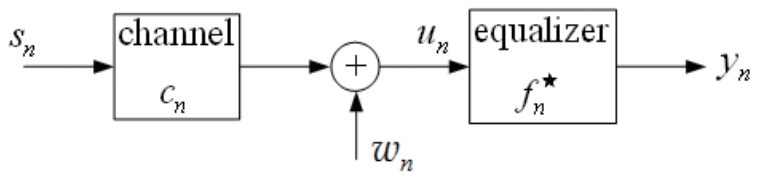

Fig. 1. A complex basedband-equivalent model.

The constant modulus algorithm (CMA) is one of the most widely used blind equalization algorithms [1]-[3]. CMA is known to be phase-independent and one way to deal with its phase ambiguity is through the use of a carrier phase rotator to produce the correct constellation orientation, which increases the complexity of the implementation of the receiver. Moreover, for high-order quadrature amplitude modulation (QAM) signal constellations (especially for cross constellations such as 128-QAM, in which the corner points containing significant phase information are not available), both the large adaptation noise and the increased sensitivity 
to phase jitter may make the phase rotator spin due to the crowded signal constellations [10]-[13]. Wesolowski [4], [5] Oh and Chin [6], and Yang, Werner and Dumont [7], proposed the multimodulus algorithm (MMA), whose cost function is given by

$$
J_{M M A}=E\left\{\left[y_{R, n}^{2}-R_{2, R}\right]^{2}\right\}+E\left\{\left[y_{I, n}^{2}-R_{2, I}\right]^{2}\right\}
$$

where $y_{R, n}$ and $y_{I, n}$ are the real and imaginary parts of the equalizer output, respectively, while $R_{2, R}$ and $R_{2, I}$ are given by $R_{2, R}=E\left[s_{R, n}^{4}\right] / E\left[s_{R, n}^{2}\right]$ and $R_{2, I}=E\left[s_{I, n}^{4}\right] / E\left[s_{I, n}^{2}\right]$, in which $s_{R, n}$ and $s_{I, n}$ denote the real and imaginary parts of $s_{n}$, respectively. Decomposing the cost function of the MMA into real and imaginary parts thus allows both the modulus and the phase of the equalizer output to be considered; therefore, joint blind equalization and carrier phase recovery may be simultaneously accomplished, eliminating the need for an adaptive phase rotator to perform separate constellation phase recovery in steady-state operation. The tap-weight vector of the MMA, $\mathbf{f}_{n}$, is updated according to the stochastic gradient descent (SGD) to obtain the blind equalizer output $y_{n}=\mathbf{f}_{n}^{H} \mathbf{u}_{n}$

$$
\mathbf{f}_{n+1}=\mathbf{f}_{n}-\mu \cdot \nabla J_{M M A}=\mathbf{f}_{n}-\mu \cdot \frac{\partial J_{M M A}}{\partial \mathbf{f}_{n}^{\star}}=\mathbf{f}_{n}-\mu \cdot e_{n}^{\star} \cdot \mathbf{u}_{n}
$$

where $\mathbf{u}_{n}=\left[u_{n+l}, \ldots, u_{n-l}\right]^{T}$ and $e_{n}=e_{R, n}+j e_{I, n}$ in which $e_{R, n}=y_{R, n} \cdot\left[y_{R, n}^{2}-R_{2, R}\right], e_{I, n}=$ $y_{I, n} \cdot\left[y_{I, n}^{2}-R_{2, I}\right]$ and $L=2 l+1$ is the tap length of the equalizer.

The analysis in [9], which concerns only the square constellations, indicates that the MMA can remove inter-symbol interference (ISI) and simultaneously correct the phase error. However, when the transmitted symbols are drawn from a QAM constellation having an odd number of bits per symbol $\left(N=2^{2 i+1}, i=2,3, \ldots\right)$, the $N$-points constellations can be arranged into an oblong constellation [14], [15] so long as $E\left[s_{R, n}^{2}\right] \neq E\left[s_{I, n}^{2}\right]$ is satisfied. For example, Fig. 2 illustrates a 128-QAM arranged by oblong $(8 \times 16)$-QAM with the required average energy of 82 . The conventional 128-cross QAM constellations with the required average energy of 82 can be obtained from a square constellation of $12 \times 12=144$ points by removing the four outer points in each corner as illustrated in Fig. 3 [7]. Notably, the distance between two adjacent message points in the oblong constellations illustrated in Fig. 2 has been modified to be 1.759 instead of 2 as in the conventional cross 128-QAM, so that the average energies required by both cross and oblong constellations are almost identical. In this chapter, the oblong constellation illustrated in Fig. 2 is used as an example to demonstrate that the MMA using asymmetric oblong QAM constellations with an odd number of bits per symbol may significantly outperform its cross counterpart in the recovery of the carrier phase introduced by channels, without requiring additional average transmitted power. We use the term asymmetric because the oblong QAM is not quadrantally symmetric, i.e., $E\left[s_{R, n}^{2}\right] \neq E\left[s_{I, n}^{2}\right]$, and as a consequence $E\left[s_{n}^{2}\right] \neq 0$. Although reducing the distance between adjacent message points in the proposed oblong constellation in Fig. 2 may increase the steady-state symbol-error rate (SER) or mean-squared error (MSE) of the adaptive equalizer, this chapter is concerned with the unique feature of fast carrier phase recovery associated with the MMA using oblong constellations during blind equalization process owing to its non-identical nature of the real and imaginary parts of the source statistics.

\section{Analysis of MMA using oblong constellations}

This section presents an analysis of the MMA using oblong QAM constellations from the perspective of its stationary points. Our analytical results demonstrate that the four saddle 


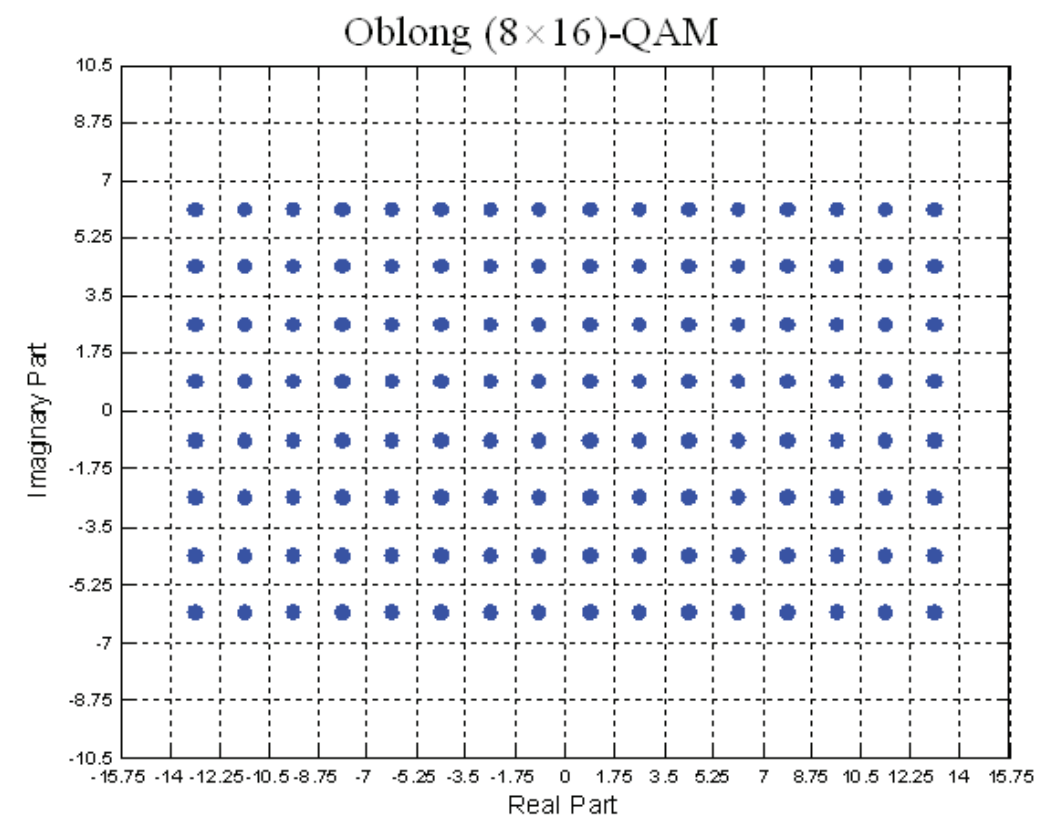

Fig. 2. Oblong constellations for 128-QAM sources.

points existing in the square and cross constellations along $\theta(k)=\pi / 4,3 \pi / 4,5 \pi / 4,7 \pi / 4$ are absent when using oblong constellations. Consequently, the frequency of being attracted towards the vicinity of the saddle points, around which the MMA exhibits slow convergence, before converging to the desired minimum, is significantly reduced when using oblong constellations. The use of oblong constellations may thus accelerate the magnitude equalization process during the transient operation.

\subsection{MMA Cost function of oblong constellations}

After some algebraic manipulation, the expansion of the MMA cost function in (1) for a complex i.i.d. zero-mean QAM source (for square, cross, and oblong constellations) with each member of the symbol alphabet being equiprobable, and a complex baseband channel excluding additive channel noise, can be written in the combined channel-equalizer space $\mathbf{h}_{n}$ as

$$
\begin{gathered}
J_{M M A}=\frac{1}{4} \cdot \Re\left\{E\left[s^{4}\right] \cdot \sum_{i} h^{4}(i)+3 E^{2}\left[s^{2}\right] \cdot \sum_{i} \sum_{l \neq i} h^{2}(i) h^{2}(l)\right\}+\frac{3}{4}\left(k_{s} \sigma_{s}^{4} \cdot \sum_{i}|h(i)|^{4}\right. \\
\left.+2 \sigma_{s}^{4} \sum_{i} \sum_{l \neq i}|h(i)|^{2}|h(l)|^{2}+E^{2}\left[s^{2}\right] \cdot \sum_{i} \sum_{l \neq i} h^{2}(i)\left(h^{2}(l)\right)^{*}\right)-R_{2, R} \cdot\left(\Re\left\{E\left[s^{2}\right] \cdot \sum_{i} h^{2}(i)\right\}\right. \\
\left.+\sigma_{s}^{2} \cdot \sum_{i}|h(i)|^{2}\right)-R_{2, I} \cdot\left(-\Re\left\{E\left[s^{2}\right] \cdot \sum_{i} h^{2}(i)\right\}+\sigma_{s}^{2} \cdot \sum_{i}|h(i)|^{2}\right)+R_{2, R}^{2}+R_{2, I}^{2}
\end{gathered}
$$

where $\sigma_{s}^{2}=E\left[\left|s_{n}\right|^{2}\right]=E\left[s_{R, n}^{2}\right]+E\left[s_{I, n}^{2}\right]$ and $k_{s}=E\left[\left|s_{n}\right|^{4}\right] / \sigma_{s}^{4}$ is the source kurtosis. The first term of (3), (1/4) $\Re\left\{E\left[s^{4}\right] \cdot \sum_{i} h^{4}(i)\right\}$, which is related to the fourth-order statistics (or 


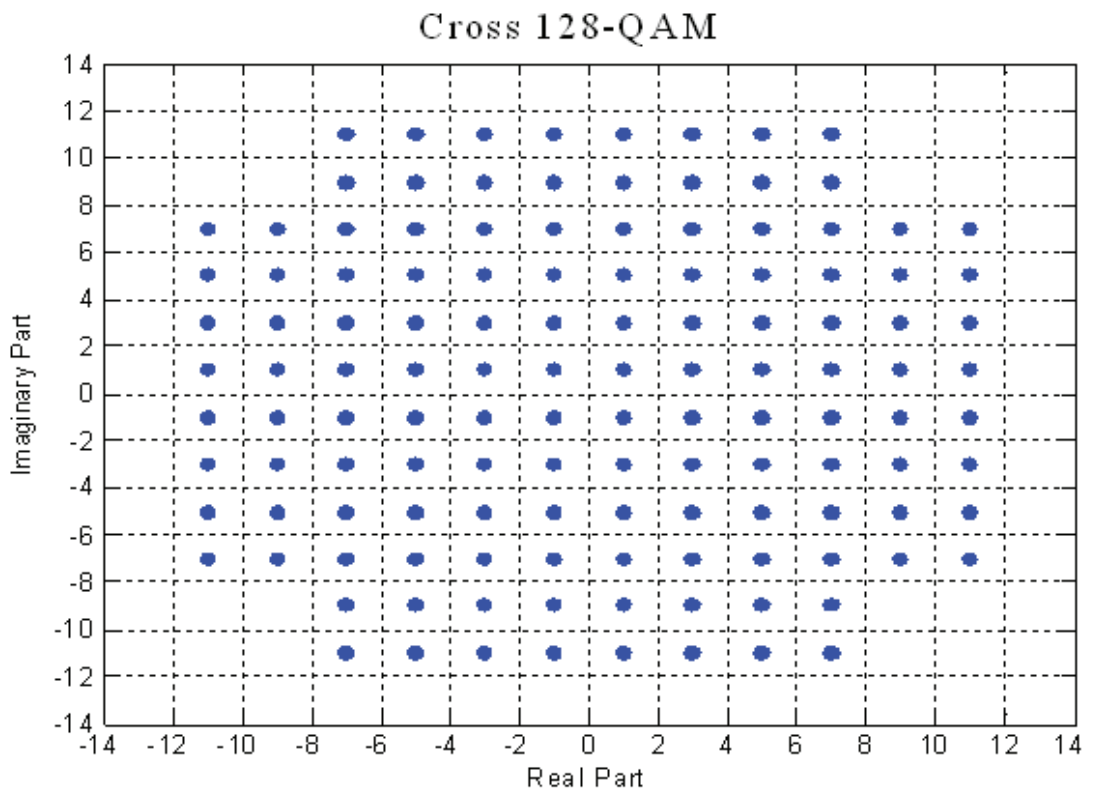

Fig. 3. cross constellations for 128-QAM sources.

fourth-power phase estimator) in [12], [13], [16]-[18] containing the phase information and is absent from the CMA cost function, allows the MMA to recover a possible phase rotation of the equalizer output. Note that when there is no possibility of confusion, the notation is simplified by suppressing the time index $n$, so for example, $s_{n} \triangleq s=s_{R}+j s_{I}$ and $h_{n}(k) \triangleq h(k)$. Terms $s_{R}$ and $s_{I}$ are assumed to be uncorrelated, and both are zero-mean, sub-Gaussian (such that $\left.E\left[|s|^{4}\right]-2 E^{2}\left[|s|^{2}\right]-\left|E\left[s^{2}\right]\right|^{2}<0\right)$. The values of the source statistics of, for example, the oblong $(8 \times 16)$-QAM can be computed to be $E\left[s_{R}^{2}\right]=65.08$ and $E\left[s_{I}^{2}\right]=16.08$. For convenience of mathematical analysis, (3) can also be expressed in the following polar space with $[r(k), \theta(k)]=\left[\sqrt{h_{R}^{2}(k)+h_{I}^{2}(k)}, \tan ^{-1}\left(h_{I}(k) / h_{R}(k)\right)\right]$, where $h(k)=h_{R}(k)+j h_{I}(k)$,

$$
\begin{aligned}
& J_{M M A}=\frac{1}{4} E\left[s^{4}\right] \cdot \sum_{i} r^{4}(i) \cos 4 \theta(i)+\frac{3}{2} \cdot E^{2}\left[s^{2}\right] \cdot\left(\sum_{i} \sum_{l \neq i} r^{2}(i) r^{2}(l) \cos [2 \theta(i)] \cos [2 \theta(l)]\right) \\
& +\frac{3}{4} \cdot k_{s} \sigma_{s}^{4} \sum_{i} r^{4}(i)+\frac{3}{2} \cdot \sigma_{s}^{4} \sum_{i} \sum_{l \neq i} r^{2}(i) r^{2}(l)-R_{2, R} \cdot\left[\left(E\left[s_{R}^{2}\right]-E\left[s_{I}^{2}\right]\right) \cdot \sum_{i} r^{2}(i) \cos 2 \theta(i)\right. \\
& \left.+\sigma_{s}^{2} \cdot \sum_{i} r^{2}(i)\right]-R_{2, I} \cdot\left[-\left(E\left[s_{R}^{2}\right]-E\left[s_{I}^{2}\right]\right) \cdot \sum_{i} r^{2}(i) \cos 2 \theta(i)+\sigma_{s}^{2} \cdot \sum_{i} r^{2}(i)\right]+R_{2, R}^{2}+R_{2, I}^{2}
\end{aligned}
$$

Notably, for oblong constellations, $R_{2, R} \neq R_{2, I}, E\left[s_{R}^{4}\right] \neq E\left[s_{I}^{4}\right], E\left[s_{R}^{2}\right] \neq E\left[s_{I}^{2}\right]$ and $E^{2}\left[s^{2}\right]=$ $E^{2}\left[s_{R}^{2}\right]+E^{2}\left[s_{I}^{2}\right]-2 E\left[s_{R}^{2}\right] E\left[s_{I}^{2}\right] \neq 0$. This asymmetric nature makes the major difference between an oblong constellation and a square (or cross) constellation, since the shape of their resulting MMA cost surfaces would be significantly different, as is revealed later in this chapter. 


\subsection{Stationary points of MMA using oblong constellations}

The equalizer is assumed to be either doubly-infinite in length as in [8] or of finite-length fractionally spaced as in [3] under the equalizability conditions. The set of stationary points of the MMA for oblong constellations considering $M \geq 1$ can be obtained by setting the gradient of $J_{M M A}$ in (4) to zero, such that $\nabla J_{M M A}=\mathbf{r} \frac{\partial J_{M M A}}{\partial r(k)}+\frac{\Theta}{r(k)} \frac{\partial J_{M M A}}{\partial \theta(k)}=0$. The components of the gradients are

$$
\begin{gathered}
\frac{\partial J_{M M A}}{\partial r(k)}=E\left[s^{4}\right] \cdot r^{3}(k) \cdot \cos 4 \theta(k)+6 E^{2}\left[s^{2}\right] \cdot r(k) \cdot \cos 2 \theta(k) \cdot\left(\sum_{l \neq k} r^{2}(l) \cdot \cos 2 \theta(l)\right) \\
+3 k_{s} \cdot \sigma_{s}^{4} \cdot r^{3}(k)+6 r(k) \cdot \sigma_{s}^{4} \cdot \sum_{l \neq k} r^{2}(l)-2 R_{2, R} \cdot r(k) \cdot\left\{\left[\left(E\left[s_{R}^{2}\right]-E\left[s_{I}^{2}\right]\right)\right.\right. \\
\left.\quad \cdot \cos 2 \theta(k)]+\sigma_{s}^{2}\right\}-2 R_{2, I} \cdot r(k) \cdot\left\{-\left[\left(E\left[s_{R}^{2}\right]-E\left[s_{I}^{2}\right]\right) \cdot \cos 2 \theta(k)\right]+\sigma_{s}^{2}\right\} \\
\frac{\partial J_{M M A}}{\partial \theta(k)}=-E\left[s^{4}\right] \cdot[\sin 4 \theta(k)] \cdot r^{4}(k)-6^{2}\left[s^{2}\right] \cdot r^{2}(k) \cdot[\sin 2 \theta(k)] \cdot\left(\sum_{l \neq k} r^{2}(l) \cdot \cos 2 \theta(l)\right) \\
+2 \sin 2 \theta(k) \cdot\left(E\left[s_{R}^{2}\right]-E\left[s_{I}^{2}\right]\right) \cdot\left(R_{2, R}-R_{2, I}\right) \cdot r^{2}(k)
\end{gathered}
$$

where we set each to zero. Clearly, one stationary point is at $r(k)=0$. For $r(k)>0$, (6) yields both $\sin 4 \theta(k)=0$ and $\sin 2 \theta(k)=0$ or

$$
\theta(k)=\frac{n \pi}{2},(n=0,1,2,3)
$$

which reveals the location of the four stationary points of using the oblong constellations. This is in contrast to the square (or cross) constellations, for which $R_{2, R}=R_{2, I}$ and $E\left[s_{R}^{2}\right]=E\left[s_{I}^{2}\right]$ such that the last two terms of (6) are both zero. Therefore, only $\sin 4 \theta(k)=0$ is required and, consequently,

$$
\theta(k)=\frac{n \pi}{4},(n=0, \ldots, 7)
$$

The four stationary points given in (7) are now located. Substituting $\theta(k) \in\{0, \pi\}$ and $\theta(l) \in$ $\{0, \pi\}$ (such that $\cos 2 \theta(k)=\cos 2 \theta(l)=1$ ) into (5) yields

$$
4 r_{+}^{2}(k)\left(E\left[s_{R}^{4}\right]+E\left[s_{I}^{4}\right]\right)+12\left(E^{2}\left[s_{R}^{2}\right]+E^{2}\left[s_{I}^{2}\right]\right) \cdot \sum_{l \neq k} r_{+}^{2}(l)-4 E\left[s_{R}^{4}\right]-4 E\left[s_{I}^{4}\right]=0, k=1, \ldots, M
$$

where we have used $E\left[s^{4}\right]=E\left[s_{R}^{4}\right]+E\left[s_{I}^{4}\right]-6 E\left[s_{R}^{2}\right] \cdot E\left[s_{I}^{2}\right]$. Similarly, substituting $\theta(k) \in$ $\{\pi / 2,3 \pi / 2\}$ and $\theta(l) \in\{\pi / 2,3 \pi / 2\}$ (such that $\cos 2 \theta(k)=\cos 2 \theta(l)=-1$ ) into (5) yields

$$
4 r_{-}^{2}(k)\left(E\left[s_{R}^{4}\right]+E\left[s_{I}^{4}\right]\right)+12\left(E^{2}\left[s_{R}^{2}\right]+E^{2}\left[s_{I}^{2}\right]\right) \cdot \sum_{l \neq k} r_{-}^{2}(l)-4 R_{2, R} \cdot E\left[s_{I}^{2}\right]-4 R_{2, I} \cdot E\left[s_{R}^{2}\right]=0, k=1, \ldots, M
$$

Clearly, (9) and (10) give $r_{+}^{2}(1)=r_{+}^{2}(2)=\ldots=r_{+}^{2}(M)$ and $r_{-}^{2}(1)=r_{-}^{2}(2)=\ldots=r_{-}^{2}(M)$, respectively. Consequently, (9) and (10) given $k=M$ suffice to determine $r_{+}^{2}(M)$ and $r_{-}^{2}(M)$ :

$$
\begin{aligned}
& r_{+}^{2}(M)=\frac{E\left[s_{R}^{4}\right]+E\left[s_{I}^{4}\right]}{E\left[s_{R}^{4}\right]+E\left[s_{I}^{4}\right]+[3(M-1)] \cdot\left(E^{2}\left[s_{R}^{2}\right]+E^{2}\left[s_{I}^{2}\right]\right)} \\
& r_{-}^{2}(M)=\frac{R_{2, R} \cdot E\left[s_{I}^{2}\right]+R_{2, I} \cdot E\left[s_{R}^{2}\right]}{E\left[s_{R}^{4}\right]+E\left[s_{I}^{4}\right]+[3(M-1)] \cdot\left(E^{2}\left[s_{R}^{2}\right]+E^{2}\left[s_{I}^{2}\right]\right)}
\end{aligned}
$$


The following form for the four stationary points along $\theta(k)=0, \pi / 2, \pi, 3 \pi / 2$ for each of the $M$ non-zero components of $\mathbf{h}_{n}$ (for oblong constellations only) can thus be derived

$$
|h(k)|^{2}=\left[r_{+}^{2}(M) \sum_{i \in I_{n}, \theta(k) \in\{0, \pi\}} \delta(k-i)\right]+\left[r_{-}^{2}(M) \sum_{i \in I_{n}, \theta(k) \in\left\{\frac{\pi}{2}, \frac{3 \pi}{2}\right\}} \delta(k-i)\right]
$$

Notably, since the four stationary points for each element of $\mathbf{h}_{n}$ have precisely the same location in the $\left[h_{R}(k), h_{I}(k)\right]$ plane for a given $\mathrm{M}$, the four stationary points given in (13) may be viewed as the stationary points in terms of the overall vector $\mathbf{h}_{n}$ in the MMA cost in (3) under the common $h_{R}(k)$ and $h_{I}(k)$ space denoting the real and imaginary parts of $h(k)$.

\subsection{Two special cases: Square and cross constellations}

For the special cases of both square and cross constellations, i.e., $R_{2, R}=R_{2, I}, E\left[s_{R}^{2}\right]=E\left[s_{I}^{2}\right]$ and $E\left[s_{R}^{4}\right]=E\left[s_{I}^{4}\right]$, (4) reduces to the following cost function

$$
\begin{aligned}
J_{M M A} & =\frac{1}{4} E\left[s^{4}\right] \cdot \sum_{i} r^{4}(i) \cos 4 \theta(i)+\frac{3}{4}\left[k_{s} \sigma_{s}^{4} \sum_{i} r^{4}(i)+2 \sigma_{s}^{4} \sum_{i} \sum_{l \neq i} r^{2}(i) r^{2}(l)\right] \\
& -2 \sigma_{S}^{2} \cdot R_{2, R} \cdot \sum_{i} r^{2}(i)+2\left(R_{2, R}\right)^{2}
\end{aligned}
$$

It has been shown in [9] that the following form for all possible stationary points of the MMA (for square and cross constellations), except for $r(k)=0$, can be expressed as

$$
\left|h_{M}(k)\right|^{2}=\left[r_{ \pm}^{2}(M) \sum_{i \in I_{n}, \theta(k) \in\left\{0, \frac{\pi}{2}, \pi, \frac{3 \pi}{2}\right\}} \delta(k-i)\right]+\left[r_{\times}^{2}(M) \sum_{i \in I_{n}, \theta(k) \in\left\{\frac{\pi}{4}, \frac{3 \pi}{4}, \frac{5 \pi}{4}, \frac{7 \pi}{4}\right\}} \delta(k-i)\right]
$$

where $r_{ \pm}^{2}(M)=E\left[s_{R}^{4}\right] /\left(E\left[s_{R}^{4}\right]+[3(M-1)] \cdot E^{2}\left[s_{R}^{2}\right]\right)$ and $r_{\times}^{2}(M)=2 E\left[s_{R}^{4}\right] /\left(E\left[s_{R}^{4}\right]+[3(2 M-\right.$ $\left.1)] \cdot E^{2}\left[s_{R}^{2}\right]\right)$. Figure 4 depicts the MMA cost surface for a cross $128-Q A M$ input for $M=1$. Notably, both $r_{+}^{2}(M)$ in (11) and $r_{-}^{2}(M)$ in (12) reduce to $r_{ \pm}^{2}(M)$ when $E\left[s_{R}^{2}\right]=E\left[s_{I}^{2}\right]$ and $E\left[s_{R}^{4}\right]=E\left[s_{I}^{4}\right]$.

\subsection{Unstable equilibria of MMA when $M \geq 2$}

If the distribution of $s_{n}$ is sub-Gaussian, then all the pre-specified $\mathbf{h}_{n}$ (with the associated $I_{M}$ ) with the stationary points shown in (13), for $M \geq 2$, can be shown to be unstable equilibria (saddle points) by applying the concept proposed by Foschini [8]. Consequently, all the vectors $\mathbf{h}_{n}, M=2,3, \ldots$, are saddle points. The locations of the four stationary points at $\left[ \pm r_{+}(M), 0\right]$ and $\left[0, \pm r_{-}(M)\right]$ for $\mathbf{h}_{n}$ with the $(8 \times 16)-Q A M$ source for different $M$ can be computed by using (11) and (12). For example, $r_{+}(10)=0.249, r_{-}(10)=0.17 ; r_{+}(5)=0.36$, $r_{-}(5)=0.245 ; r_{+}(2)=0.611, r_{-}(2)=0.416 ; r_{+}(1)=1, r_{-}(1)=0.68$. Clearly, the four saddle points are located nearer the origin as the number of non-zero components of $\mathbf{h}_{n}$ rises. As $M$ decreases during the blind equalization process, the locations of the four saddle points for $\mathbf{h}_{n}$ move dynamically away from the origin in four mutually perpendicular directions. The four saddle points eventually converge as $M \rightarrow 1$ to $[ \pm 1,0]$ and $\left[0, \pm r_{-}(1)\right]$, where the former two stationary points become the only two global minima, and the latter two are still two saddle points.

When compared with (8) and (15), the result in (7) and (13) is significant, since it implies that, for $M \geq 2$ (during the transient (or startup) mode operation), the number of saddle points is only half those of the square and cross constellations when the oblong constellations 


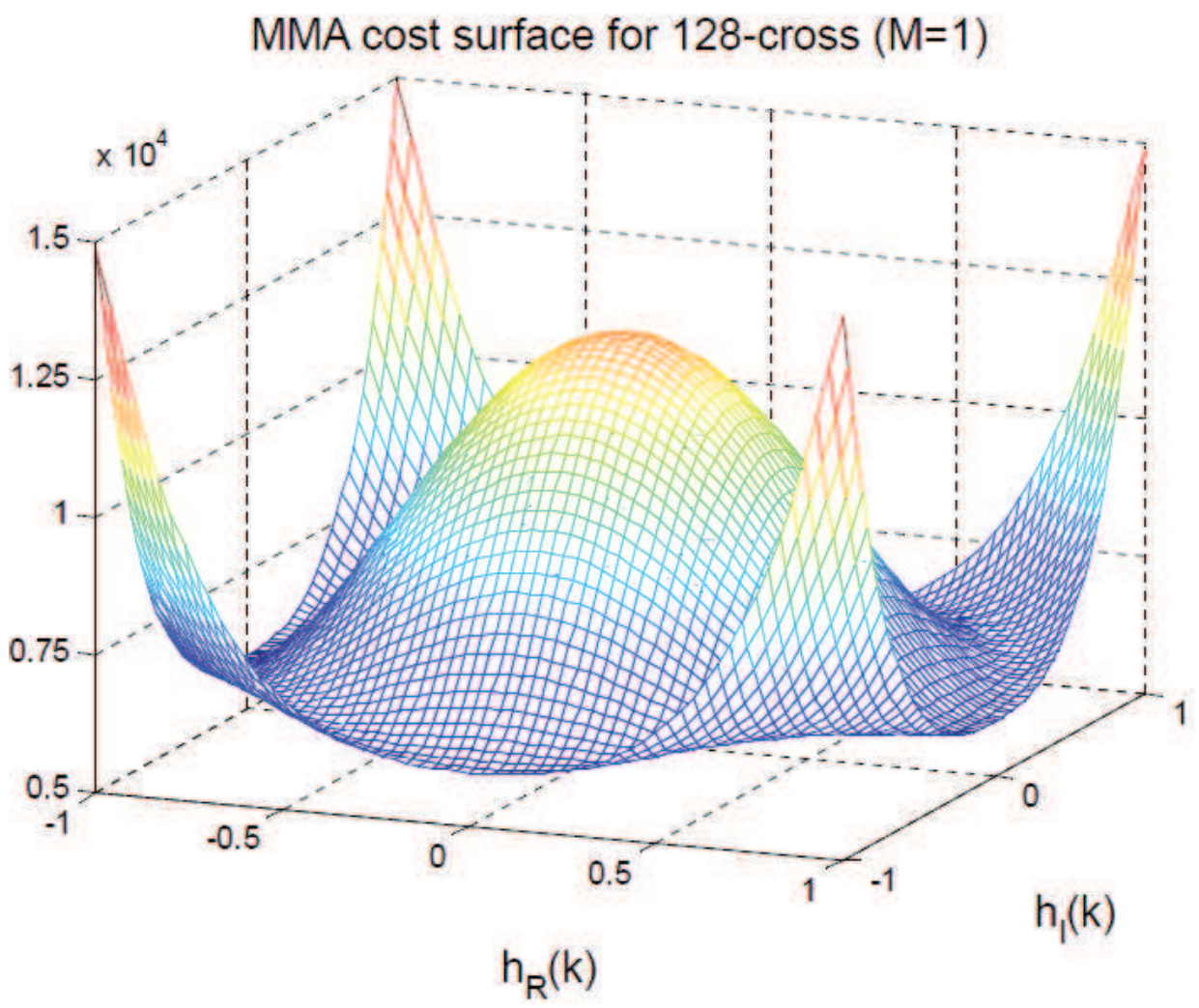

Fig. 4. MMA cost surface for 128-cross input as a function of $h_{R}(k)$ and $h_{I}(k)$ for $M=1$

are adopted (i.e., the four saddle points existing in square and cross constellations along $\theta(k)=\pi / 4,3 \pi / 4,5 \pi / 4,7 \pi / 4$ disappear). Consequently, the frequency of attraction toward the vicinity of the saddle points, around which it exhibits slow convergence, before converging to the desired minimum, is significantly diminished when using oblong constellations. Accordingly, using oblong constellations may accelerate the magnitude equalization (or residual ISI removing) process during the transient mode operation.

2.5 Local maximum and local minimum of MMA when $M \geq 2$

Computer simulations performed in this study demonstrate that once blind equalization started, a non-zero component of $\mathbf{h}_{n}$ with maximum magnitude square, $r^{2}(k)$, rose, and the sum of the magnitude squared of the remaining $M-1$ nonzero components, $\sum_{l \neq k} r^{2}(l)$, fell rapidly, eventually diminishing to zero. The non-zero component of $\mathbf{h}_{n}$ with maximum magnitude square is called " $h(k)$ with maximum modulus" in the remainder of this chapter. This chapter focuses on the MMA cost in terms of $h(k)$ with maximum modulus, which indicates the performance of the MMA during the transient mode operation $(M \geq 2)$. To discover how $h(k)$ with maximum modulus evolves during the transient operation when the MMA adopts the oblong QAM based on the SGD, the MMA cost can be considered in terms of $h(k)$ with maximum modulus alone by substituting the approximations $\sum_{k} \sum_{l \neq k} r^{2}(k) r^{2}(l) \cong 0$, 
$\sum_{l} r^{4}(l) \cong r^{4}(k)$, and $\sum_{l} r^{2}(l) \cong r^{2}(k)$ (i.e., the sum of magnitude square of the remaining $(M-1)$ non-zero components of $\mathbf{h}_{n}$ are very small once the MMA begins functioning) into (4).

From (5) and (6), one stationary point is located at the origin. The second derivative can be shown that when $M \geq 2, \nabla^{2} J_{M M A} \cong 12 \cdot k_{s} \sigma_{s}^{4} \cdot r^{2}(k)-4 \cdot \sigma_{s}^{2} \cdot\left[R_{2, R}+R_{2, I}\right]$. Substituting $r^{2}(k)=0$ into the above equation, yields $\nabla^{2} J_{M M A} \cong-4 \cdot \sigma_{s}^{2} \cdot\left[R_{2, R}+R_{2, I}\right]<0$, because the situation involving $h(k)$ with maximum modulus at the origin is our concern. The origin thus corresponds to a local maximum in the MMA cost function in terms of $h(k)$ with maximum modulus alone during the transient mode operation. However, the origin corresponds to a local minimum in the MMA cost function in terms of $h(l), l \neq k$ (i.e., the remaining $M-1$ nonzero components of $\mathbf{h}_{n}$ ), during the transient mode operation, provided that $\nabla^{2} J_{M M A}>0$ at the origin $\left(\right.$ or $\left.r^{2}(k)>\left[R_{2, R}+R_{2, I}\right] \cdot \sigma_{S}^{2} / 3 k_{s} \sigma_{S}^{4} \cong 0.3868\right)$ for oblong $(8 \times 16)$-QAM input.

2.6 Desired global minima of MMA when $M=1$

When $M \rightarrow 1$ (as in steady-state mode), the combined channel-equalizer impulse response vector $\mathbf{h}_{n}=[0, \ldots, 0, h(k), 0, \ldots, 0]^{T}$ can be shown to be the only set of minima. The set of all possible stationary points of vector $\mathbf{h}_{n}$ for oblong constellations given $M=1$ can be summarized as (i) $r(k)=0$; (ii) $r_{+}(k)=1, \theta(k)=0$ or $\pi$; (iii) $r_{-}^{2}(k)=$ $\frac{R_{2, R} \cdot E\left[s_{I}^{2}\right]+R_{2, I} \cdot E\left[s_{R}^{2}\right]}{E\left[s_{R}^{4}\right]+E\left[s_{I}^{4}\right]}, \theta(k)=\pi / 2$ or $3 \pi / 2$ by substituting $M=1$ into (11) and (12). Examining the second derivative of the cost function in (4) indicates that for a sub-Gaussian input, $\left[h_{R}(k), h_{I}(k)\right]=[0,0],\left[h_{R}(k), h_{I}(k)\right]=[ \pm 1,0]$ and $\left[h_{R}(k), h_{I}(k)\right]=\left[0, \pm \sqrt{\left.\frac{R_{2, R} \cdot E\left[s_{I}^{2}\right]+R_{2, I} \cdot E\left[s_{R}^{2}\right]}{E\left[s_{R}^{4}\right]+E\left[s_{I}^{4}\right]}\right]}\right.$ correspond to a local maximum, local (hence global) minima and two saddle points, respectively. Notably, the finding that $[1,0]$ and $[-1,0]$ are the only two global minima reveals that any phase error within $180^{\circ}$ can be correctly detected when using oblong constellations. This finding contrasts with equalizer output with a $90^{\circ}$ phase ambiguity when using the quadrantally symmetric QAM square and cross constellations because phase errors that are multiples of $90^{\circ}$ are undetectable. Figure 5 depicts the MMA cost surface for oblong $(8 \times 16)-Q A M$ input with $M=1$ in (3) such that $\mathbf{h}_{n}=\left[0, \ldots, 0, h_{R}(k)+j h_{I}(k), 0, \ldots, 0\right]^{T}$.

Even for $M \geq 2$, the MMA cost function in (4) can be visualized in terms of each non-zero element of $\mathbf{h}_{n}$ having four saddle points along $\theta(k)=0, \pi / 2, \pi, 3 \pi / 2$. More specifically, there is one local maximum at the origin in terms of $h(k)$ with maximum modulus and one local minimum at the origin in terms of $h(l), l \neq k$, provided that $r^{2}(k)>0.3868$, explaining why $h(k)$ with maximum modulus moves toward $\left[ \pm r_{+}(M), 0\right]$, rather than the origin, while the remaining $M-1$ nonzero components of $\mathbf{h}_{n}$ move toward the origin, based on the SGD method, during the transient operation.

\section{Carrier phase tracking capability}

This section presents two superior phase tracking capabilities of the oblong constellation. First, when using the MMA with the oblong QAM based on the SGD, $h(k)$ with maximum modulus automatically moves toward the stationary points at $\left[r_{+}(M), 0\right]$ and $\left[-r_{+}(M), 0\right]$, respectively, when $-90^{\circ}<\theta(k)<90^{\circ}$ and $90^{\circ}<\theta(k)<270^{\circ}$, once the magnitude of $h(k)$ with maximum modulus is large enough during the transient mode operation. Meanwhile, the remaining $M-1$ nonzero components of $\mathbf{h}_{n}$ tend to diminish, and the two stationary points at $\left[r_{+}(M), 0\right] \rightarrow[1,0]$ and $\left[-r_{+}(M), 0\right] \rightarrow[-1,0]$ automatically become the only two global minima when $M \rightarrow 1$. Second, an oblong constellation permits much faster phase 
MMA cost function for oblong ( $8 \times 16)-Q A M(M=1)$

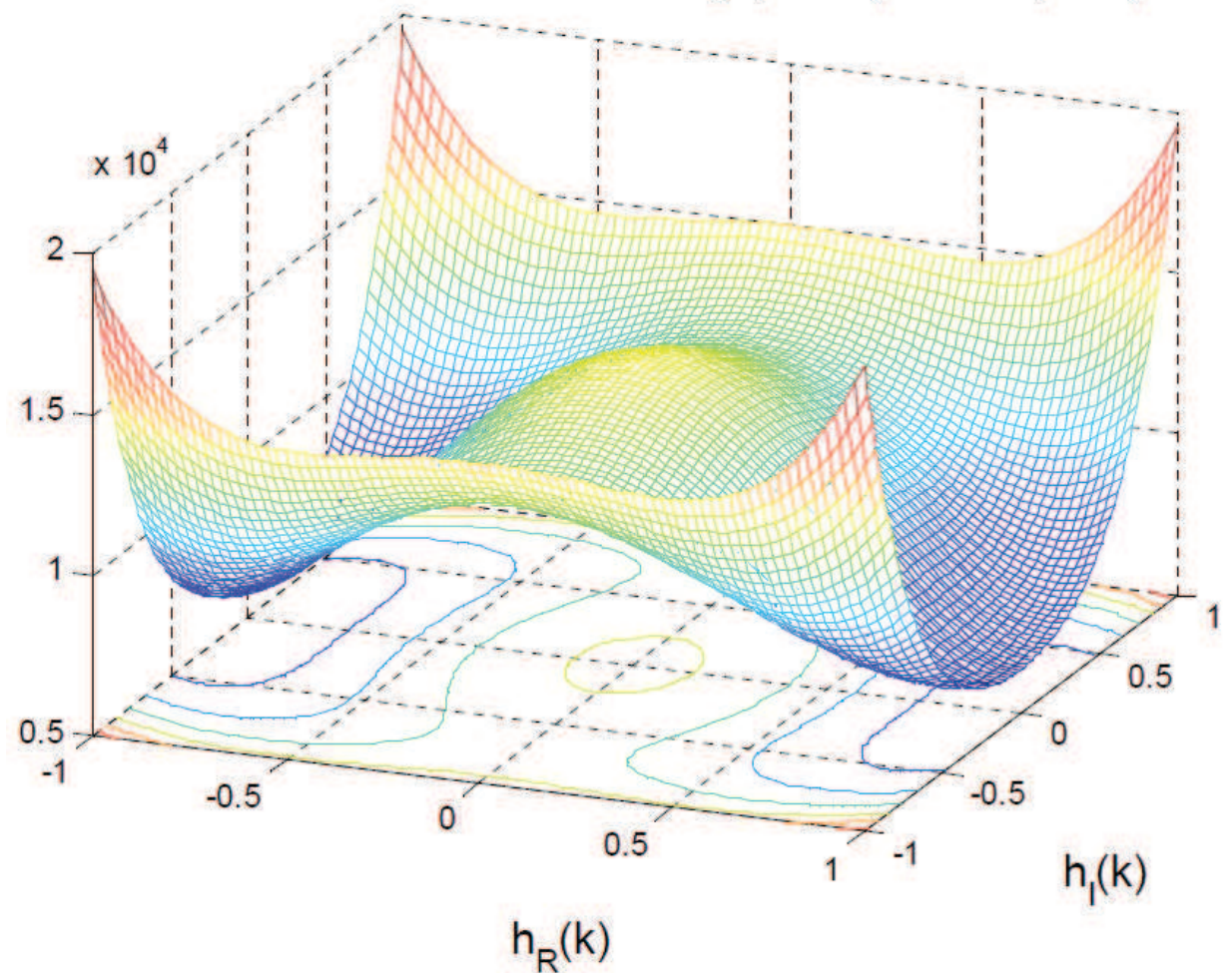

Fig. 5. MMA cost surface for $(8 \times 16)-Q A M$ input as a function of $h_{R}(k)$ and $h_{I}(k)$ for $M=1$

acquisition and tracking rates than a cross constellation, because the shape of the cost surface of the oblong constellation inherently has a much steeper average gradient toward the two global minima than the cross constellation.

\subsection{Dynamic phase tracking using $-\nabla_{\theta} J_{M M A}$}

We start by rewriting (6) as $\frac{\partial J_{M M A}}{\partial \theta}=2 \sin 2 \theta(k)\left\{F \cdot r^{2}(k)-A \cdot r^{4}(k) \cos 2 \theta(k)-3 B \cdot r^{2}(k)\right.$. $\left.\sum_{l \neq k} r^{2}(l) \cdot \cos 2 \theta(l)\right\}$, where, for notational simplicity, $A=E\left[s^{4}\right], B=E^{2}\left[s^{2}\right]$, and $F=$ $\left\{E\left[s_{R}^{2}\right]-E\left[s_{I}^{2}\right]\right\} \cdot\left[R_{2, R}-R_{2, I}\right]$. For the special cases of square and cross constellations, $F=0$ and $B=0$ leading to $\nabla_{\theta}=\frac{\Theta}{r(k)} \frac{\partial J_{M M A}}{\partial \theta(k)}=-E\left[s^{4}\right] \cdot r^{3}(k) \cdot \sin 4 \theta(k)$, which is exactly the result shown in [9] for the square QAM during the transient operation. This section is mainly concerned with the magnitude of $h(k)$ with maximum modulus [i.e., $r(k)=\max (|h(k)|)]$ and its corresponding phase $\theta(k)=\tan ^{-1} \frac{h_{I}(k)}{h_{R}(k)}$. If $C=\left\{F \cdot r^{2}(k)-A \cdot r^{4}(k) \cos 2 \theta(k)-3 B \cdot r^{2}(k)\right.$. $\left.\sum_{l \neq k} r^{2}(l) \cdot \cos 2 \theta(l)\right\}>0$ can be proved, then the sign of $\frac{\partial J_{M M A}}{\partial \theta(k)}=2 \sin 2 \theta(k) \cdot C$ is determined entirely by $\sin 2 \theta(k)$. Hence, the non-zero component of $\mathbf{h}_{n}$ with maximum modulus based on the SGD in (2) can be readily shown to move toward the stationary points along $\theta(k)=0$ and 
$\theta(k)=180^{\circ}$, respectively, when $-90^{\circ}<\theta(k)<90^{\circ}$ and $90^{\circ}<\theta(k)<270^{\circ}$, as shown in Fig. 6(a), provided that $C>0$.

We consider the strictest condition when $\cos 2 \theta(l)=1$, for $l \neq k$ and, consequently, $C$ may be rewritten as $C=\left\{F \cdot r^{2}(k)-A \cdot r^{4}(k) \cos 2 \theta(k)-3 B \cdot r^{2}(k) \cdot \sum_{l \neq k} r^{2}(l)\right\}$. In order to obtain the condition of the size of $r^{2}(k)$ required for $C>0$ [see (16) below], $E\left[|y|^{2}\right] \cong E\left[|s|^{2}\right]$ was assumed to be satisfied (i.e., magnitude equalization was successfully accomplished with the constant power constraint). Consequently, $\sum_{l \neq k} r^{2}(l) \cong 1-r^{2}(k)$ was obtained by using $E\left[|y|^{2}\right]=$ $E\left[|s|^{2}\right] \cdot \sum_{l} r^{2}(l)$ [30]. This assumption is justifiable because one unique feature of the MMA is that its magnitude equalization and carrier phase recovery remain simultaneously effective once it starts functioning. However, this carrier phase recovery takes much longer than the magnitude equalization, which might be almost accomplished in fewer than 1500 iterations, in contrast to 10000 iterations or even more (e.g., for 128-QAM) for the carrier phase recovery. Consequently, the MMA might still produce a high SER, because the equalizer output still exhibits a large phase rotation even once the magnitude equalization has almost been accomplished (ISI $\leq-20 d B$ ), where ISI is defined as $\operatorname{ISI}(d B)=10 \log \left\{\frac{\sum_{k}|h(k)|^{2}-\max \left(|h(k)|^{2}\right)}{\max \left(|h(k)|^{2}\right)}\right\}$. By using the approximation, $\sum_{l \neq k} r^{2}(l) \cong 1-r^{2}(k)$, it is straightforward to show that the condition for $C>0$ is

$$
r^{2}(k)=h_{R}^{2}(k)+h_{I}^{2}(k)>\frac{3 B-F}{3 B-A \cdot \cos 2 \theta(k)}
$$

Consequently, the MMA using the oblong QAM based on the SGD moves toward $\theta(k)=0^{\circ}$ and $\theta(k)=180^{\circ}$ when $-90^{\circ}<\theta(k)<90^{\circ}$ and $90^{\circ}<\theta(k)<270^{\circ}$, respectively. Equation (16) reveals that the carrier phase recovery, and the tracking of any frequency offset of the MMA using the oblong QAM, hinges on the success of magnitude equalization. To give a rough idea of the size of $r^{2}(k)$ required to satisfy (16), thus allowing carrier phase recovery to be undertaken and allowing the equalizer to be switched to the decision-directed mode, let $\theta(k) \rightarrow 0$ (or $\theta(k) \rightarrow \pi$ ) such that the blind equalizer is transitioning to phase recovery regime. Then $\cos 2 \theta(k)$ approximates unity and (16) becomes $r^{2}(k) \cong h_{R}^{2}(k)>$ $(3 B-F) /(3 B-A)$, which equals around 0.54 for $(8 \times 16)-Q A M$ input. The condition in (16) becomes increasingly strict as $\theta(k) \rightarrow 0$ (or $\theta(k) \rightarrow \pi$ ). For example, if $\theta(k)=\pi / 6$, then $r^{2}(k)>0.459$ for the $(8 \times 16)-Q A M$ input. Simulation results confirm that the condition in (16) is almost immediately satisfied as long as the channel is well equalized by choosing an appropriate step-size parameter. By contrast, for the square and cross constellations, $h(k)$ with maximum modulus unconditionally moves toward the four stationary points at $\left[ \pm r_{ \pm}(M), 0\right]$ or $\left[0, \pm r_{ \pm}(M)\right]$, as long as $E\left[s^{4}\right]<0$, which is valid for most sub-Gaussian source (other than 8-PSK, 4-PSK, and V.29 modem [28]), as shown in Fig. 6(b), since for these two special cases, $F=0, B=0$ and $A<0$, (16) becomes $r^{2}(k)>0$, which is naturally satisfied. Also note that the narrower the constellations (e.g., $(4 \times 32)-Q A M$ input) is used, the condition in (16) becomes stricter. As an example, for the $(4 \times 32)-Q A M$ input, $(16)$ becomes $r^{2}(k)>0.95$ when $\theta(k) \rightarrow 0$. This result reveals that the use of a very narrow oblong constellation may not yield satisfactory phase tracking capability.

\subsection{Fast carrier phase recovery}

The average slope (i.e., the average gradient) of the MMA cost surface using oblong constellation, in terms of $h(k)$ with maximum modulus alone, from saddle point $\left[0, r_{-}(M)\right]$ toward $\left[r_{+}(M), 0\right]$, was compared with that of the cross constellation from saddle point 


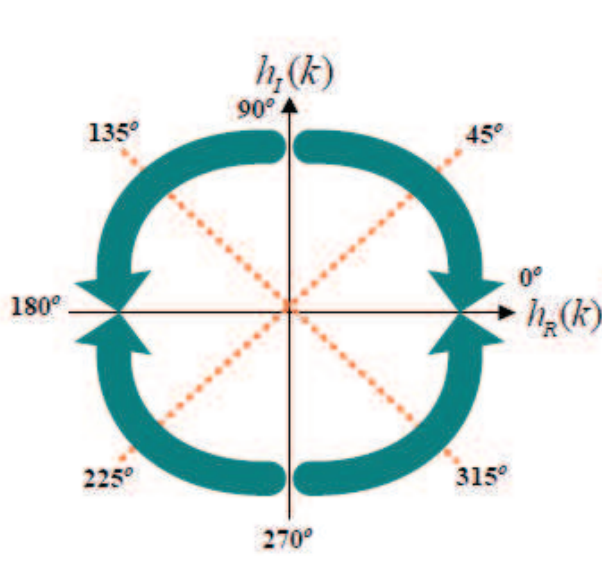

(a)

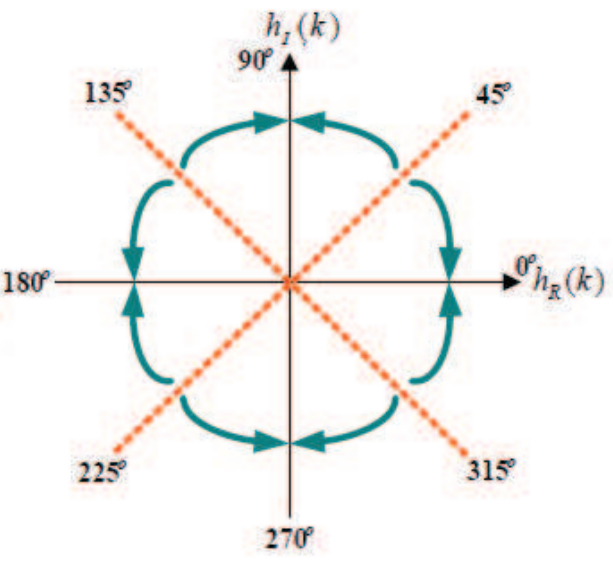

(b)

Fig. 6. Trajectory of $h(k)$ with maximum modulus using SGD for (a) oblong QAM and (b) square and cross QAM.

$(1 / \sqrt{2}) \cdot\left[r_{\times}(M), r_{\times}(M)\right]$ toward $\left[r_{ \pm}(M), 0\right]$ in order to compare their phase tracking speeds. This is because the rate of phase tracking hinges on the diminishing rate of the imaginary part of $h(k)$ with maximum modulus, which eventually diminishes to zero when the phase rotation is fully recovered. Notably, due to the $180^{\circ}$ symmetric QAM oblong constellation and the quadrantally symmetric QAM cross constellations, exactly the same results would have been obtained using the slope from the other saddle point $\left[0,-r_{-}(M)\right]$ to $\left[ \pm r_{+}(M), 0\right]$ for the oblong constellation, and the slope from $(1 / \sqrt{2}) \cdot\left[ \pm r_{\times}(M), \pm r_{\times}(M)\right]$ to $\left[ \pm r_{ \pm}(M), 0\right]$ or $\left[0, \pm r_{ \pm}(M)\right]$ for the cross constellation.

The average slope between two points at $[a, b]$ and $[c, d]$ in the MMA cost surface in terms of $h(k)$ with maximum modulus alone is defined as slope $=\frac{J_{M M A}[a, b]-J_{M M A}[c, d]}{\sqrt{(a-c)^{2}+(b-d)^{2}}}$, where $J_{M M A}[a, b]$ is calculated by substituting $[a, b]$ into the MMA cost function in (4) (for oblong constellation) or in (14) (for cross constellation) by using the approximations $\sum_{l} r^{2}(l) \cong r^{2}(k), \sum_{l} r^{4}(l) \cong$ $r^{4}(k)$, and $\sum_{k} \sum_{l \neq k} r^{2}(k) r^{2}(l) \cong 0$ (i.e., the sum of magnitude square of the remaining $(M-1)$ non-zero components of $\mathbf{h}_{n}$ are very small once the MMA begins functioning). The average slope of the MMA cost from $\left[0, r_{-}(M)\right]$ to $\left[r_{+}(M), 0\right]$ for the oblong constellation and the average slope of the MMA cost from $(1 / \sqrt{2}) \cdot\left[r_{\times}(M), r_{\times}(M)\right]$ to $\left[r_{ \pm}(M), 0\right]$ for the cross constellation can be approximated as, respectively,

$$
\text { slope }_{o b l}=\frac{J_{M M A}\left[0, r_{-}(M)\right]-J_{M M A}\left[r_{+}(M), 0\right]}{\sqrt{r_{+}^{2}(M)+r_{-}^{2}(M)}}=\frac{A}{\sqrt{r_{+}^{2}(M)+r_{-}^{2}(M)}}
$$

and

$$
\text { slope }_{\text {cross }}=\frac{J_{M M A}\left[\frac{r_{\times}(M)}{\sqrt{2}}, \frac{r_{\times}(M)}{\sqrt{2}}\right]-J_{M M A}\left[r_{ \pm}(M), 0\right]}{\sqrt{\left[\frac{r_{\times}(M)}{\sqrt{2}}-r_{ \pm}(M)\right]^{2}+\left[\frac{r_{x}(M)}{\sqrt{2}}-0\right]^{2}}}
$$




$$
=\frac{B}{\sqrt{r_{\times}^{2}(M)+r_{ \pm}^{2}(M)-\sqrt{2} \cdot r_{ \pm}(M) \cdot r_{\times}(M)}}
$$

where $A=(-1 / 4) \cdot E\left[s^{4}\right] \cdot\left[r_{+}^{4}(M)-r_{-}^{4}(M)\right]+(3 / 4) \cdot k_{s} \sigma_{s}^{4} \cdot\left[r_{-}^{4}(M)-r_{+}^{4}(M)\right]-\left[R_{2, R}+R_{2, I}\right] \cdot$ $\sigma_{s}^{2} \cdot\left[r_{-}^{2}(M)-r_{+}^{2}(M)\right]+\left[E\left[s_{R}^{2}\right]-E\left[s_{I}^{2}\right]\right] \cdot\left[R_{2, R}-R_{2, I}\right] \cdot\left[r_{-}^{2}(M)+r_{+}^{2}(M)\right]$ and $B=(-1 / 4)$. $E\left[s^{4}\right] \cdot\left[r_{\times}^{4}(M)+r_{ \pm}^{4}(M)\right]+(3 / 4) \cdot k_{s} \sigma_{s}^{4} \cdot\left[r_{\times}^{4}(M)-r_{ \pm}^{4}(M)\right]-2 \cdot R_{2, R} \cdot \sigma_{s}^{2} \cdot\left[r_{\times}^{2}(M)-r_{ \pm}^{2}(M)\right]$. Comparison of $A$ with $B$ term by term indicates that the first three terms of both $\mathrm{A}$ and $\mathrm{B}$ are similar. However, the dominating term, which is the last term in A, contributes to the large average slope of the MMA cost surface of using the oblong constellation, owing to its asymmetric nature of $E\left[s_{R}^{2}\right]>E\left[s_{I}^{2}\right]$ and $R_{2, R}>R_{2, I}$ inherent in the oblong constellation. The ratio between the slopes of the two MMA cost surfaces can then be defined as $r=\frac{\text { slope }_{\text {obl }}}{\text { slope }_{\text {cross }}}=$ $\frac{A \cdot \sqrt{r_{\times}^{2}(M)+r_{ \pm}^{2}(M)-\sqrt{2} \cdot r_{ \pm}(M) \cdot r_{\times}(M)}}{B \cdot \sqrt{r_{+}^{2}(M)+r_{-}^{2}(M)}}$, which is calculated as 3.1, 7.6, 12.56, 17.54 and 25.02 for $M=1,3,5,7$ and 10 , respectively, when comparing oblong $(8 \times 16)-Q A M$ with cross $128-$ $Q A M$. The imaginary part of $h(k)$ with maximum modulus using oblong constellation would therefore diminish to zero much faster than that using cross constellation. Consequently, when using oblong QAM based on the SGD, $h(k)$ with maximum modulus moves toward the two global minima at $[ \pm 1,0]$ much more rapidly than that of using the cross constellation. Notably, the MMA cost surface of oblong constellation slopes down more sharply than its cross counterpart as the number of non-zero components of $\mathbf{h}_{n}$ rises. This finding reveals that the imaginary part of $h(k)$ with maximum modulus of oblong constellation diminishes much faster than that of using the cross constellation, especially during the startup operation of blind equalization. Also note that the larger the difference between $E\left[s_{R}^{2}\right]$ and $E\left[s_{I}^{2}\right]$ for a particular oblong constellation, the steeper the average gradient of the cost surface of oblong constellation toward global minima than that of cross constellation.

\section{Computer simulations}

Some blind carrier phase tracking algorithms [20]-[23] and some multimodulus-based algorithms [24], [25] for cross-QAM signals have been developed recently. Computer simulations were performed to compare the performance of the MMA using both oblong and cross constellations, and the performance of CMA using cross constellations followed by either a dispersion minimization derotator (DMD) [20] or a phase tracker (PT) [21], [22] [29] to correct the carrier phase offset of the CMA output. The computer simulations also included a sliced constant cross algorithm (SCXA) for joint blind equalization and phase recovery of odd-bit cross QAM proposed by Abrar and Qureshi [24]. The transmitted data symbol $s_{n}$ is an i.i.d., and may be one of the following four possible sources: conventional 128-cross, oblong $(8 \times 16)$-QAM , and two modified 128-QAM constellations proposed by Cartwright [23], known as the $128 \mathrm{~A}-\mathrm{COB}$ and $128 \mathrm{C}-\mathrm{COB}$, whose variance of the fourth power phase estimator is much smaller than that of the 128-cross. The average energies of the conventional 128-cross, $(8 \times 16)$-oblong , $128 \mathrm{~A}-\mathrm{COB}$, and $128 \mathrm{C}-\mathrm{COB}$, are all 82 , while the peak-to-average energy ratios for the four constellations are 2.073, 2.585, 2.645, and 2.881, respectively. Notably, the distance between two adjacent message points in 128A-COB and 128C-COB has been reduced to 1.893 and 1.976, respectively, instead of 2 . The channel used for the simulations was the Brazil Ensemble C [26]. Computer experiments were carried out by using the baseband-equivalent channel model developed in [27] with fractionally spaced equalizers (FSE), and the sampling frequency was chosen to be four times the symbol rate. The input 
to each sub-equalizer is a $40^{\circ}$ phase-rotated version of $u_{n}^{(j)}=\sum_{i} c_{i}^{(j)} s_{n-i}+w_{n}^{(j)}, j=1,2,3,4$. The real and imaginary parts of the complex-valued additive white Gaussian noise $w_{n}^{(j)}$ were assumed to be independent, and had equal variance such that the signal-noise-ratios (SNRs) were $30 \mathrm{~dB}$. Here, $S N R=P_{a v g} / 2 \sigma_{w}^{2}$ in which $P_{a v g}$ is the average power of the signal constellation and $\sigma_{w}^{2}$ is the variance of each component of the complex valued noise source. In the FSE, each of the four sub-equalizers had 31 complex tap weights with 15 units of time delay. The tap weights of the fourth sub-equalizer were initialized by setting the central tap weight to 1 and the others to zero, while all the tap weights of the remaining three sub-equalizers were set to zero. The ensemble-averaged SER over 10 independent runs was used as a performance index shown in Fig. 7 in which the symbol duration is $0.093 \mu$ s as in the ATSC DTV standard.

The MMA using $(8 \times 16)$ - oblong displayed a faster convergence rate than that of $C M A+$ $D M D, C M A+P T, S C X A, M M A$, using the conventional 128-cross and CMA+PT using both $128 A$-COB and $128 C$-COB, according to Fig. 7 . However, the MMA using $(8 \times 16)$-oblong yielded a higher steady-state SER than that of $C M A+P T$ and $C M A+D M D$ using the conventional 128-cross because the use of oblong constellations generates a higher steady-state MSE than its conventional cross counterpart. Notably, the use of MMA without requiring a phase rotator such as DMD and PT reduces the complexity of the implementation of the receiver. Moreover, both DMD and PT yield results with a possible ambiguity of $90^{\circ}$ when 128-cross, 128A-COB, and 128C-COB are used. Therefore, the use of MMA with oblong constellations reduces the number of possible phase ambiguities by half. The $C M A+P T$ using the conventional 128-cross outperformed the CMA+PT using 128A-COB and 128C-COB because the latter two constellations whose kurtosis $k_{s}=1.462$ and $k_{s}=1.399$, respectively, are larger than that of the 128-cross (with $k_{s}=1.34$ ), sacrifice some equalizer convergence rate (i.e., residual ISI removal speed) in order to gain some phase tracking speed, especially when the ISI of the channel is severe. Simulation results similar to those of Fig. 7 were also obtained when the same channel with different phase rotations were adopted because the carrier phase offset was overshadowed by the severity of the ISI of the channel. However, the simulation results also show that the SCXA proposed in [24] outperformed all the other schemes when the phase rotations were less than $30^{\circ}$ because the SCXA sometimes became trapped in some undesirable local minima when the phase rotations were greater than $30^{\circ}$.

\section{Conclusion}

The analysis in this chapter demonstrates that the MMA using oblong constellations is less likely to be attracted to saddle points than the MMA using both square and cross constellations because the number of saddle points is only half of those of the square and cross constellations when adopting oblong constellations during the transient mode operation. Moreover, the finding that $[1,0]$ and $[-1,0]$ are the only two global minima reveals that any phase error within $180^{\circ}$ can be correctly detected when using oblong constellations. The $180^{\circ}$ phase ambiguity (and the $90^{\circ}$ phase ambiguity for 128 -cross, $128 \mathrm{~A}-\mathrm{COB}$, and $128 \mathrm{C}-\mathrm{COB}$ ) may be overcome by using differential encoding when necessary. This chapter also shows that the oblong constellations to be used with the MMA may outperform the conventional cross constellations in terms of carrier phase recovery owing to $E\left[s_{R}^{2}\right] \neq E\left[s_{I}^{2}\right]$. Intuitively, this may be because the MMA cost function given by (1) reduces to $J_{M M A}=2 E\left\{\left[y_{R, n}^{2}-R_{2, R}\right]^{2}\right\}$, $J_{M M A}=2 E\left\{\left[y_{R, n}^{2}-R_{2, R}\right]\right\}$ which essentially considers only the real part of the equalizer output, when the symmetric constellations are such that $R_{2, R}=R_{2, I}, E\left[s_{R}^{4}\right]=E\left[s_{I}^{4}\right]$ and 


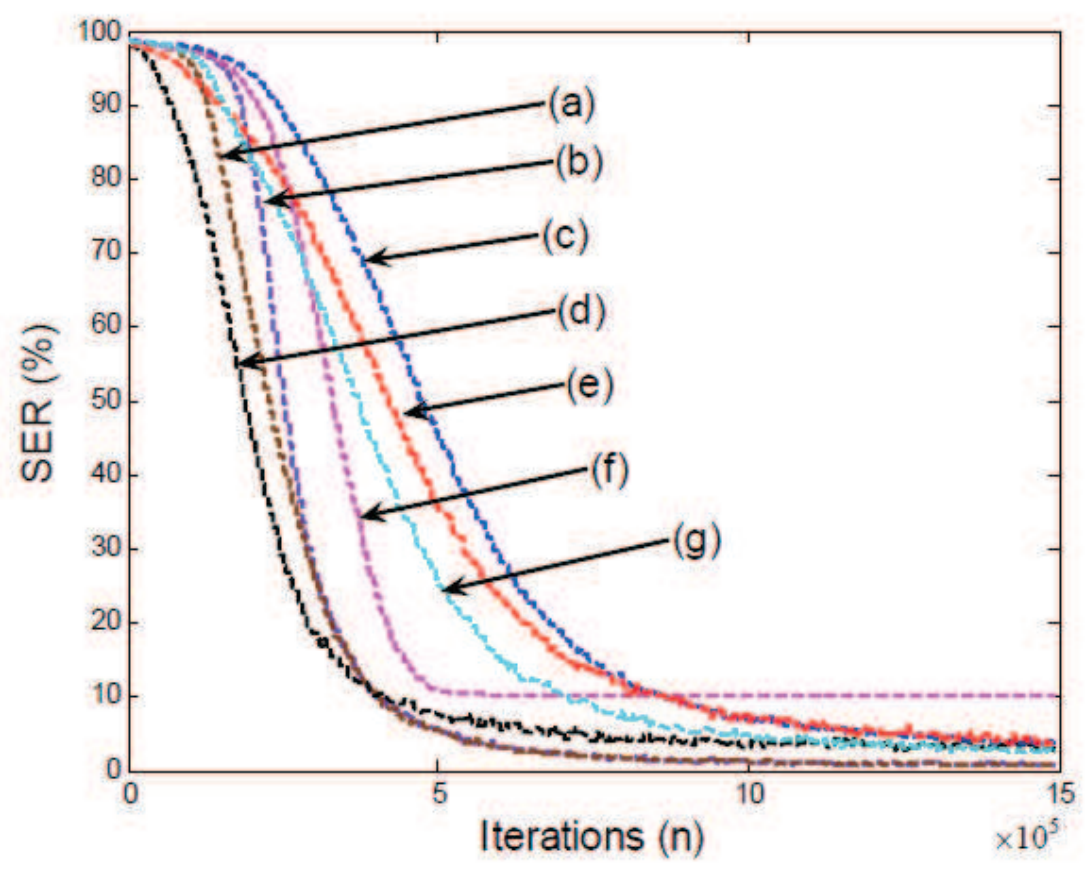

Fig. 7. Ensemble-averaged SER performance using the Brazil Ensemble $C$ for $S N R=30 d B$ and phase rotation $40^{\circ}$. Traces: (a) CMA+PT using 128-cross $\left(\mu=9 \times 10^{-9}, \mu_{P T}=2 \times 10^{-8}\right)$, (b) CMA $+D M D$ using 128-cross

$\left(\mu=9 \times 10^{-9}, \mu_{D M D}=4 \times 10^{-8}\right)$, (c) $M M A$ using 128-cross $\left(\mu=6 \times 10^{-9}\right),(\mathrm{d})$ MMA using oblong $(8 \times 16)$-QAM $\left(\mu=10^{-8}\right)$, (e) CMA + PT using $128 A$-COB $\left(\mu=6 \times 10^{-9}, \mu_{P T}=5 \times 10^{-8}\right)$, (f) SCXA using 128-cross $\left(\mu=3 \times 10^{-9}\right)$, and $(\mathrm{g})$ CMA + PT using $128 C-\mathrm{COB}\left(\mu=6 \times 10^{-9}, \mu_{P T}=10^{-8}\right)$.

$E\left[s_{R}^{2}\right]=E\left[s_{I}^{2}\right]$. From a statistical perspective, the MMA cost function in (1) can be exploited fully for blind equalization only when the asymmetric nature of the constellations is utilized. However, the oblong constellation has a slightly higher peak-to-average power ratio and a higher steady-state SER than the conventional cross constellation at the same average energy level. Other asymmetric constellations may be designed (such as by adjusting the symbol probabilities without reducing the distance between adjacent message points) to achieve the best compromise among fast carrier phase recovery, low steady-state SER and low average transmitted power, as long as $E\left[s_{R}^{2}\right] \neq E\left[s_{I}^{2}\right]$.

\section{References}

[1] D. N. Godard (1980). "Self-recovering equalization and carrier tracking in two-dimensional data communication system," IEEE Trans. Commun., vol. COM-28, pp.1867-1875, Nov. 1980. 
[2] J. R. Treichler and M. G. Larimore (1985). "New Processing Techniques Based on the Constant Modulus Algorithm," IEEE Trans. Acoust., Speech, Signal Process., vol. ASSP-33, pp.420-431, Apr. 1985.

[3] C. R. Johnson, Jr., P. Schniter, T. J. Endres, J. D. Behm, D. R. Brown, and R. A. Casas (1998). "Blind equalization using the constant modulus criterion: A review," Proc. IEEE, vol. 86, pp.1927-1950, Oct. 1998.

[4] K. Wesolowski (1987). "Self-recovering adaptive equalization algorithms for digital radio and voiceband data modems," Proc. European Conf. Circuit Theory and Design, 1987, pp. 19-24.

[5] K. Wesolowski (1992). "Analysis and properties of the modified constant modulus algorithm for blind equalization," European Trans. Telecommun., vol. 3, pp. 225-230, May-June 1992.

[6] K. N. Oh and Y. O. Chin (1995). "Modified constant modulus algorithm: blind equalization and carrier phase recovery algorithm," Proc. IEEE Int. Conf. Commun., 1995, vol. 1, pp. 498-502.

[7] J. Yang, J.-J. Werner, and G. A. Dumont (2002). "The multimodulus blind equalization and its generalized algorithms," IEEE J. Sel. Areas Commun., vol. 20, no. 5, pp. 997-1015, Jun. 2002.

[8] G. J. Foschini (1985). "Equalization without altering or detecting data," AT\&T Tech. J., vol. 64, pp. 1885-1911, Oct. 1985.

[9] J.-T. Yuan and K.-D. Tsai (2005). "Analysis of the Multimodulus Blind equalization Algorithm in QAM Communication Systems," IEEE Trans. Commun., Vol. 53, No. 9, pp. 1427-1431, Sep. 2005.

[10] N. K. Jablon (1992). "Joint blind equalization, carrier recovery, and timing recovery for high-order QAM signal constellations," IEEE Trans. Signal Process., vol.40, no. 6, pp.1383-1397, Jun. 1992.

[11] J.-T. Yuan and L.-W. Chang (2007). "Carrier phase tracking of multimodulus blind equalization algorithm using QAM oblong constellations," Proc. IEEE Int. Conf. Commun., Glasgow, Scotland, UK, 2007, pp. 2991-2998.

[12] C. N. Georghiades (1997). "Blind carrier phase acquisition for QAM constellations," IEEE Trans. Commun., vol. 45, pp. 1477-1486, Nov. 1997.

[13] E. Serpedin, P. Ciblat, G.. B. Giannakis, and P. Loubaton (2001). "Performance analysis of blind carrier phase estimators for general QAM constellations," IEEE Trans. Signal Process., vol. 49, no. 8, pp. 1816-1823, Aug. 2001.

[14] J. G. Smith (1975). "Odd-bit quadrature amplitude-shift keying," IEEE Trans. Commun., vol. 23, pp. 385-389, Mar. 1975.

[15] K. Cho and D. Yoon (2002). "On the general BER expression of one- and two-dimensional amplitude modulations," IEEE Trans. Commun., vol. 50, pp. 1074-1080, Jul. 2002.

[16] M. Moeneclaey and G. de Jonghe (1994). "ML-oriented NDA carrier synchronization for general rotationally symmetric signal constellations," IEEE Trans., Commun., vol. 42, Aug. 1994, pp. 2531-2533.

[17] K. V. Cartwright (1999). "Blind phase recovery in general QAM communication systems using alternative higher order statistics," IEEE Signal Processing Lett., vol. 6, no. 12, Dec. 1999, pp. 327-329.

[18] R. Lopez-Valcarce (2004). "Cost minimization interpretation of fourth power phase estimator and links to multimodulus algorithm," Electron. Lett., vol. 40, no. 4, Feb. 2004. 
[19] J. P. LeBlanc, I. Fijalkow, and C. R. Johnson, Jr. (1998). "CMA fractionally spaced equalizers: stationary points and stability under iid and temporally correlated sources," Int. J. Adapt. Control Signal Process., vol. 12, pp. 135-155, 1998.

[20] W. Chung, W. A. Sethares, and C. R. Johnson, Jr. (2004). "Performance analysis of blind adaptive phase offset correction based on dispersion minimization," IEEE Trans. Signal Process., vol.52, no. 6, pp.1750-1759, Jun. 2004.

[21] H. Mathis (2001). "Blind phase synchronization for VSB signals," IEEE Trans. on Broadcast., vol.47, no. 4, pp.340-347, Dec. 2001.

[22] S. Abrar (2007). "An adaptive method for blind carrier phase recovery in a QAM receiver," in Proc. Int. Conf. on Information Emerging Technologies, 2007, pp. 1-6.

[23] K. V. Cartwright (2000). "Fourth power phase estimation with alternative two-dimensional odd-bit constellations," IEEE Commun. Lett., vol. 4, pp. 199-201, Jun. 2000.

[24] S. Abrar and I. M. Qureshi (2006). "Blind equalization of cross-QAM signals," IEEE Signal Processing Lett, vol. 13, no. 12, pp. 745-748, Dec. 2006.

[25] S. Chanmanon and T. Thaiupathump (2006). "Performance analysis of double-ellipse algorithm for QAM equalization and phase recovery," 2006 Int. Symposium on Commun. and Inf. Technologies, pp. 657-662.

[26] C. W. Rhodes (2002). "Some recent improvements in the design of DTV receivers for the ATSC standard," IEEE Trans. Consumer Electron., vol. 48, pp. 938-945, Nov. 2002.

[27] H. -N. Kim, Y. -T. Lee, and S. W. Kim (2003). "Mathematical modeling of VSB-based digital television systems," ETRI J., vol. 25, pp. 9-18, Feb. 2003.

[28] J.-T. Yuan and T.-C. Lin (2010). "Equalization and Carrier Phase Recovery of CMA and MMA in Blind Adaptive Receivers," IEEE Trans. Signal Process., vol. 58, no.6, pp. 3206-3217, Jun. 2010.

[29] J.-T. Yuan and Y.-F. Huang (2010). "Blind Carrier Phase Acquisition and Tracking for 8-VSB Signals," IEEE Trans. Commun., vol. 58, no. 3, pp. 769-774, Mar. 2010.

[30] O. Shalvi and E. Weinstein (1990). "New criteria for blind deconvolution of nonminimum phase systems (channels)," IEEE Trans. Inf. Theory, vol. 36, pp. 312-321, Mar. 1990. 


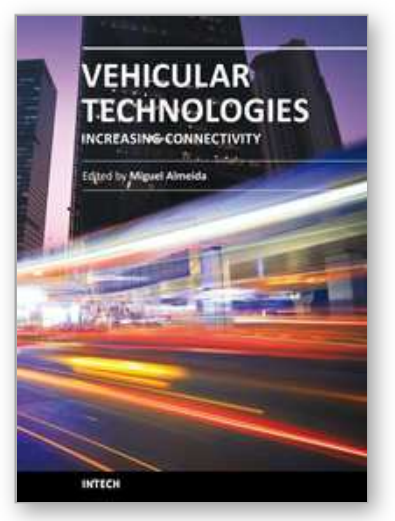

\author{
Vehicular Technologies: Increasing Connectivity \\ Edited by Dr Miguel Almeida
}

ISBN 978-953-307-223-4

Hard cover, 448 pages

Publisher InTech

Published online 11, April, 2011

Published in print edition April, 2011

This book provides an insight on both the challenges and the technological solutions of several approaches, which allow connecting vehicles between each other and with the network. It underlines the trends on networking capabilities and their issues, further focusing on the MAC and Physical layer challenges. Ranging from the advances on radio access technologies to intelligent mechanisms deployed to enhance cooperative communications, cognitive radio and multiple antenna systems have been given particular highlight.

\title{
How to reference
}

In order to correctly reference this scholarly work, feel free to copy and paste the following:

Jenq-Tay Yuan and Tzu-Chao Lin (2011). Multimodulus Blind Equalization Algorithm Using Oblong QAM Constellations for Fast Carrier Phase Recovery, Vehicular Technologies: Increasing Connectivity, Dr Miguel Almeida (Ed.), ISBN: 978-953-307-223-4, InTech, Available from: http://www.intechopen.com/books/vehiculartechnologies-increasing-connectivity/multimodulus-blind-equalization-algorithm-using-oblong-qamconstellations-for-fast-carrier-phase-rec

\section{INTECH}

open science | open minds

\section{InTech Europe}

University Campus STeP Ri

Slavka Krautzeka 83/A

51000 Rijeka, Croatia

Phone: +385 (51) 770447

Fax: +385 (51) 686166

www.intechopen.com

\section{InTech China}

Unit 405, Office Block, Hotel Equatorial Shanghai

No.65, Yan An Road (West), Shanghai, 200040, China

中国上海市延安西路65号上海国际贵都大饭店办公楼 405 单元

Phone: +86-21-62489820

Fax: +86-21-62489821 
(C) 2011 The Author(s). Licensee IntechOpen. This chapter is distributed under the terms of the Creative Commons Attribution-NonCommercialShareAlike-3.0 License, which permits use, distribution and reproduction for non-commercial purposes, provided the original is properly cited and derivative works building on this content are distributed under the same license. 